\title{
The roles of reconstruction and lexical storage in the comprehension of regular pronunciation variants
}

\author{
Mirjam Ernestus ${ }^{1,2}$ \\ ${ }^{1}$ Center for Language Studies, Radboud University, Nijmegen, The Netherlands \\ ${ }^{2}$ Max Planck Institute for Psycholinguistics, Nijmegen, The Netherlands \\ Mirjam.Ernestus@mpi.nl
}

\begin{abstract}
This paper investigates how listeners process regular pronunciation variants, resulting from simple general reduction processes. Study 1 shows that when listeners are presented with new words, they store the pronunciation variants presented to them, whether these are unreduced or reduced. Listeners thus store information on word-specific pronunciation variation Study 2 suggests that if participants are presented with regularly reduced pronunciations, they also reconstruct and store the corresponding unreduced pronunciations. These unreduced pronunciations apparently have special status. Together the results support hybrid models of speech processing, assuming roles for both exemplars and abstract representations.

Index Terms: pronunciation variation, speech comprehension, lexical storage, phonological reconstruction, speech reduction,
\end{abstract} word learning

\section{Introduction}

Recent research has shown that pronunciation variation is highly abundant in conversational speech. Especially speech reduction, the articulatory weakening or complete deletion of segments and syllables, may result in many pronunciation variants for the same word. For instance, the Dutch word natuurlijk 'of course' may be produced as [natyrlək] (unreduced form), [natylək], [ntylək], [ntyk], [tyrlək] (which also occurs in written text), [tylok], [tylk], [tyk], [tyg], [dyk], and [dyg], among others [1]. The abundance of within-speaker pronunciation variation raises the question of how listeners process such variation.

The literature focuses on two very different mechanisms. Upon hearing a reduced pronunciation, listeners may reconstruct the corresponding unreduced pronunciation by means of rules or constraints. For instance, English listener may reconstruct [mis] to /mist/ because they know that wordfinal $[\mathrm{t}]$ may be absent after [s]. The reconstructed forms are mapped onto the unreduced pronunciation variants stored in the mental lexicon [2].

Alternatively, listeners may base themselves on lexical representations for all pronunciation variants. The menta lexicon of an English speaker may not only contain the pronunciation $/ \mathrm{mist} /$ for mist but also $/ \mathrm{mis} /$. Listeners may map the incoming acoustic signal onto any of the stored pronunciation variants [3].

Possibly, both mechanisms play roles in speech processing Reconstruction explains how listeners understand pronunciation variants they have not heard before but which follow general patterns, observed also for other words. The storage of pronunciation variants explains the possibility that certain (high frequency) words may show word-specific pronunciation variation, such as the pronunciation gonna for English going to. Furthermore, a role for stored pronunciation variants is likely since several studies show that listeners may remember characteristics of the speaker's voice [4,5], and therefore do not abstract away pronunciation variation. Moreover, other studies suggest that listeners may use the stored pronunciation variants resulting from simple categorical alternations, such as flapping of /nt/ clusters in American English [6].

The present paper investigates the contributions of the two mechanisms in the comprehension of pronunciation variation resulting from a simple gradient reduction process: the shortening of schwa leading to completely absent schwas. The study compares the processing of word tokens with and without schwa.
Evidence for a possible role of stored pronunciation variants would be the existence of word-specific variation, since such variation can only be accounted for by word-specific specification of the possible pronunciations. Word-specific variation is, however, difficult to ascertain, since every wordspecific variation may in fact result from some more general processes that happen to have escaped the researcher's attention.

More convincing evidence would therefore consist of experimental data showing that listeners are sensitive to wordspecific variation that is artificially introduced by the researcher. The storage of pronunciation variants predicts that if listeners are presented with new words in a reduced pronunciation variant, they store these reduced variants, even though the corresponding unreduced variants can easily be deduced. As a consequence, listeners could show sensitivity to which words showed reduction and which did not.

The present study tested which forms listeners store in two word-learning studies. In both studies native speakers of Dutch learned non-existing past-participles. In line with Dutch regular morphology, these past-participles consisted of the prefix ge$/ x \partial /$ and ended in [t]. In Dutch, the schwa of the prefix is often reduced, resulting in shortened and absent vowels. In both studies, participants learned half of the new past-participles with the unreduced prefix ([xə]) and the other half with the extremely reduced prefix $[\mathrm{x}]$. Since Dutch listeners are familiar with the pronunciation of $/ \mathrm{x} \partial /$ as $[\mathrm{x}]$, they should be able to reconstruct the unreduced pronunciation variants for the reduced words. Moreover, reconstruction was stimulated by the initial obstruent cluster of the reduced forms: The prefix was always followed by an obstruent-intial stem, and deletion of the schwa in ge- therefore resulted in phonotactically illegal obstruent clusters, which is a cue to the presence of schwa in the unreduced forms of the words. Listeners' storage of the new past-participles was tested by means of a lexical decision task.

In Study 1, the lexical decision experiment contained all new past-participles from the familiarization phase in their reduced forms. If listeners react more quickly to the words that they heard as reduced also in the familiarization phase, we may conclude that they stored the pronunciation variants that they heard in the familiarization phase, even though they could have reconstructed the unreduced pronunciations. Participants are then sensitive to word-specific variation (i.e., which words showed the reduction and which did not) for a completely regular and therefore general reduction process.

\section{Study 1}

\subsection{Introduction}

Study 1 consisted of three phases. In Phase 1 , the familiarization phase, participants learned the new pastparticiples, half with the unreduced prefix [xə] and half with the reduced prefix $[\mathrm{x}]$.

In Phase 2 , participants were tested on whether they had stored the new past-participles (in any form). Following [7], participants performed a lexical decision task including existing words that are of a low frequency of occurrence and direct phonological neighbors (i.e., differing in only one segment) for the newly acquired words. If listeners stored the new words, they are expected to react more slowly to their phonological neighbors, due to lexical competition, than a group of control participants who have not learned the new words. This control experiment is important in case the main experiment does not show an effect at all, since it then shows whether this may be due to how well participants learned the new words. Furthermore, this control experiment is interesting by itself, 
since it forms a replication of [7] with different words, a different language, and a different familiarization phase.

Finally, Phase 3 consisted of the lexical decision experiment testing which pronunciation variants participants had stored for the past-participles. All words were presented as reduced.

\subsection{Participants}

Thirty undergraduate students, all native speakers of Dutch, were paid to participate in all three parts of the study. Another 30 students participated just in the control lexical decision experiment.

\subsection{Materials}

\subsubsection{Familiarization phase}

Twenty-six non-existing past-participles were created in line with Dutch regular morphology and phonotactics. Their stems were monysyllabic and started with obstruents, while the complete words were direct neighbors of low-frequency existing words. For instance, the new past-participle getweept was a neighbor of gedweept 'has been a devotee of'.

These new past-participles were recorded several times by six female speakers, who spoke different regional variants of Standard Dutch. The speakers were instructed to produce unreduced and reduced versions of the words. In the reduced versions, the prefix $g e$ was produced as $[\mathrm{x}]$ and also the rest of the word was shorter than in the unreduced versions. Four tokens were selected for every speaker, and included in the familiarization phase. These tokens sounded most natural and in two of them the schwa was completely absent (reduced tokens) while in the other two the schwa was clearly present (unreduced tokens). The unreduced tokens had an average duration of $820 \mathrm{~ms}$ and the reduced tokens an average duration of $791 \mathrm{~ms}$.

Then 12 master lists were created, all containing 12 tokens of the 26 words in a random order. In each list, half of the words occurred only in their unreduced forms and half only in their reduced forms. Subsequently, the mirror images of these 12 lists were created by replacing the reduced tokens by the unreduced tokens, and vice versa. In the familiarization phase, each participant was presented with the tokens of one (master or mirror) list. Participants thus heard very different tokens of the same words (but all these tokens were either unreduced or reduced), which has been shown to facilitate learning [8].

Each word type was presented to the participants together with a pair of pictures indicating the meaning of the new past participle. The first picture showed an object in one state and the second picture the same object in a different state. The correspondence between a past-participle and a pair of pictures was random and different for each participant.

\subsubsection{Control lexical decision experiment}

The control lexical decision experiment contained the 26 low frequency phonological neighbors of the new past-participles, in addition to 219 other existing words and 184 pseudo words (many of them also inflected verb forms). All words had been produced in their unreduced forms by yet another female native speaker of Dutch. Each participant heard the words in one out of twelve randomized orders.

\subsubsection{Main lexical decision experiment}

The main lexical decision experiment contained only pastparticiples starting with the prefix $g e$-, which were all produced in a reduced form, by the same speaker who also recorded the stimuli for the control lexical decision experiment. The main lexical decision experiment included all 26 target pastparticiples presented to the participants in the familiarization phase, in addition to 241 other non-existing past-participles and 213 existing past-participles. The 26 target past-participles had an average duration of $915 \mathrm{~ms}$, and were therefore much longer than the reduced tokens presented in the familiarization phase. Each participant heard the words in one out of twelve random orders.

\subsection{Procedure}

\subsubsection{Familiarization phase}

The participants were asked to learn the 26 past-participles that were presented to them. They saw the pictures on a computer screen and heard the words over head phones, while sitting in a sound attenuated booth. Every trial started with a star in the middle of the screen, which was followed after $150 \mathrm{~ms}$ by the first picture. Then after $750 \mathrm{~ms}$, the second picture was shown for $1500 \mathrm{~ms}$ and the corresponding past participle was played. This familiarization phase lasted approximately 15 minutes.

\subsubsection{Lexical decision experiments}

The participants returned to the attenuated booth for the control experiment after a week, since the study by [7] indicated that only then new words may be incorporated in participants' mental lexicons. The main lexical decision experiment was carried out eight days after the familiarization phase.

For both experiments, participants were instructed to decide as quickly as possible whether a stimulus was an existing word of Dutch by pressing one of two buttons. The button for the "yes" response was always located in front of the participant's preferred hand. For the main lexical decision experiment, participants were additionally instructed to say "yes" also to the words that they had learned in the familiarization phase.

The participants heard the words over headphones. Each trial consisted of a star that was presented on the computer screen for $200 \mathrm{~ms}$, followed after $1 \mathrm{~ms}$ (control experiment) or after $5 \mathrm{~ms}$ (main experiment) by the auditory presentation of the stimulus. Three hundred $\mathrm{ms}$ after the participant had pressed a button, the star for the next trial appeared on the screen. Participants had to respond within $2500 \mathrm{~ms}$, otherwise a time out was registered. Latencies were measured from stimulus onset.

The control experiment contained in total 8 small breaks and lasted approximately 20 minutes. The main experiment contained three breaks and lasted 15 minutes.

\subsection{Results and discussion}

\subsubsection{Control lexical decision experiment}

The participants who had participated in the familiarization phase (the target group) were compared with those who had not (the control group). The two participants groups hardly differed in their numbers of errors for the phonological neighbors of the new past-participles presented in the familiarization phase (henceforth target words, 118 versus 111 errors), but showed important differences for the existing filler words (600 versus 358 errors) and above all for the pseudo words (1004 versus 206 errors).

For the analysis of the response latencies, the 18 time out errors (all produced by the control participants) were excluded from the dataset as well as the 205 latencies that were more than 3 standard deviation longer or shorter than the mean (1110 $\mathrm{ms}$ ). The means of the resulting 24935 response latencies are shown in Table 1.

Table 1. The mean response latencies in milliseconds for the two participants groups to the three types of stimuli.

\begin{tabular}{|l|c|c|}
\hline Stimulus type & Control participants & Target participants \\
\hline Target words & 1183 & 1246 \\
Filler words & 974 & 1034 \\
Pseudo words & 1070 & 1212 \\
\hline
\end{tabular}

For the statistical analysis, the response latencies were logged so that they showed a less skewed distribution. These logged response latencies were then analyzed by means of multi-level regression modeling [9] with participant and word as crossed random predictors. The most important fixed predictors were Participant group and whether the stimulus was a target word, a filler existing word, or a filler pseudo word (Stimulus type) and their interaction. In order to reduce the variance, I included also the logged durations of the words and the Correctness of the responses. The model was refitted after removal of nonsignificant interactions and after removal of data points more 
than 2.5 standard deviation away from their predicted values (outliers)

Importantly, in addition to main effects of Participant group $(\mathrm{F}(1,24258)=8.273, \mathrm{p}<0.01)$ and Stimulus type $(\mathrm{F}(2,24258)=$ $42.674, \mathrm{p}<0.001$ ), the model shows a statistically significant interaction between these two predictors $(\mathrm{F}(2,24258)=88.382$ $\mathrm{p}<0.0001)$. This interaction suggests that the target participants were affected by the familiarization phase.

Further analysis showed that the target participants were slower than the control participants for all word types but that the difference between the participant groups was greater for the pseudo words than for the filler existing words $(\mathrm{F}(1,22760)=144.854, \mathrm{p}<0.0001)$ and the target words $(\mathrm{F}(1,13769)=58.697, \mathrm{p}<0.0001)$. This pattern is similar to the error pattern (see above). A possible explanation is that the target participants expected the study to focus on pseudo words, since also the familiarization phase focused on pseudo words. They were therefore more careful in taking their decisions for pseudo words.

More importantly, the target and control participants were equally delayed by the (low frequent) target words compared to the filler words (a delay of $212 \mathrm{~ms}$ for the target participants and of $209 \mathrm{~ms}$ for the control participants, $\mathrm{p}>0.1$ ). This experiment thus presents no evidence that the target participants have incorporated in their mental lexicons words presented to the them in the familiarization phase.

\subsubsection{Main lexical decision experiment}

Participants produced many errors in the main lexical decision experiment. They produced 1077 incorrect versus 6063 correct responses for the filler pseudo words, and 1102 incorrect versus 5408 correct responses for the filler existing words. This high error rate is probably due to the reduction in the presented pronunciation variants. The target past-participles, which had been presented in the familiarization phase, elicited an even higher percentage of errors: 272 errors ("no"-responses) versus 508 correct ("yes") responses. This shows that the participants had difficulties judging the target past-participles as existing words of Dutch, because they did not recognize these words from the familiarization phase, or because it was unnatural for them to classify these non-existing words as existing.

The errors for the target past-participles were analyzed by means of a multi-level regression model with the binomial link function [10]. The crossed random variables were the participant and the past-participle, while the fixed predictors included the pronunciation variant of the past-participle for the given participant in the familiarization phase and the logged duration of the stimulus. None of these fixed predictors showed a significant effect (all ps $>0.1$ )

For the analysis of the response latencies, the dataset did not include the 10 latencies that differed more than three standard deviations from the mean latency $(1308 \mathrm{~ms})$. The data were analyzed again by means of a multilevel regression analysis with as dependent variable the log of the response latency, and as random effects the participant and the past participle. The fixed effect predictors were the response given by the participant ("yes" or "no"), the duration of the stimulus (again logged), and most importantly, whether the participant had learned that word in the reduced or unreduced form (familiarization type). After removal of the outliers, only familiarization type appeared significant $(\mathrm{F}(1,753)=9.745, \mathrm{p}<$ 0.01 ). Participants reacted more quickly to a reduced form in the lexical decision experiment if they had learned that same word as reduced in the familiarization phase (average response latency: $1271 \mathrm{~ms}$ ) than if they had learned that word in the unreduced form (average response latency: $1319 \mathrm{~ms}$ ).

Importantly, the results did not show an interaction between the participants' response and familiarization type Such an interaction would be expected if a "no"-response implied that the participant had not recognized the target pastparticiple. Apparently, participants mostly answered "no" because they did not consider the target past-participles as existing words of Dutch. They produced a "no" response more quickly for past-participles that they had heard as reduced before, since they then recognized them more easily.

In conclusion, the main effect of familiarization type suggests that participants stored the words in the pronunciation variants presented to them in the familiarization phase. This shows first that the participants learned the words in the familiarization phase, even though we could not find evidence for this learning in the control lexical decision experiment More importantly, the results show that listeners are sensitive to word-specific variation: They learned which words were presented as reduced and did not store all words (only) as unreduced, even though the unreduced forms could be easily derived.

The results thus support the storage of pronunciation variants and the use of these variants in speech processing. The question now arises whether participants, when presented with reduced variants, store only these reduced variants or also reconstruct and store the corresponding unreduced pronunciations. Possibly, participants, upon hearing gtweept, store both gtweept and getweept. Study 2 addressed this question.

Study 2 is identical to Study 1, except that the main lexical decision experiment contained all words in their unreduced forms. If participants stored the new past participles only in the forms in which they heard them in the familiarization phase, we expect that they react more quickly to a past-particple that they heard as unreduced also in this phase. However, if participants, upon hearing a reduced form, stored both the reduced and the unreduced forms, we expect no difference between the pastparticiples that were unreduced and those that were reduced in the familiarization phase.

\section{Study 2}

\subsection{Participants}

Thirty undergraduate students, who were all native speakers of Dutch and had not participated in Study 1, were paid to take part in Study 2. One of them could not take part in the control lexical decision experiment due to technical failures.

\subsection{Materials}

The materials were identical to those of Study 1, except that the main lexical decision experiment now contained unreduced, rather than reduced realizations of all words. These realizations were produced by the same speaker who also produced the stimuli for the other lexical decision experiments. The target past-participles in the main lexical decision had an average duration of $989 \mathrm{~ms}$ and were therefore $74 \mathrm{~ms}$ longer than the reduced tokens presented in the main lexical decision experiment of Study 1 .

\subsection{Procedure}

The procedures were identical to those of Study 1 .

\subsection{Results and discussion}

\subsubsection{Control Lexical decision experiment}

Participants produced fewer errors in this control experiment than the target participant group tested in Study 1: 75 errors for the target words, 427 for the filler existing words, and 572 for the filler pseudo words. Nevertheless, for the filler words, their numbers of errors are still much higher than those produced by the control group (see above), even though this control group contained one more participant.

The mean response latencies were 1264 for the target words, $1034 \mathrm{~ms}$ for the filler words and $1261 \mathrm{~ms}$ for the filler pseudo words. This pattern of results is very similar to the pattern produced by the target participants of Study 1 . The participants were in general slower than the control participants, and this is especially true for the pseudo words (191 ms slower for the pseudo words; $67 \mathrm{~ms}$ for the words; 81 ms for the target words).

The response latencies were analyzed as in Study 1, and again the interaction of Participant group with Stimulus type emerged as significant $(\mathrm{F}(2,23721)=7.933, \mathrm{p}<0.0001)$. Further analysis showed again that the participant groups differed more in their response latencies for the pseudo words than for the filler words $(\mathrm{F}(1,22317)=17.2692, \mathrm{p}<0.0001)$ and the target words $(\mathrm{F}(1,13346)=3.672, \mathrm{p}<0.0001)$. More importantly, there was again no statistically significant interaction between Participant group and whether the stimulus was an existing filler word or a target word. Also this experiment therefore does not show that participants had incorporated the new past participles in their mental lexicons.

\subsubsection{Main Lexical decision experiment}

Participants produced 736 incorrect and 6494 correct responses for the filler existing words, and 853 incorrect versus 5567 
correct responses for the filler pseudo words. The filler items thus elicited fewer errors than in Study 1, probably because they were reduced in Study 1 and unreduced in Study 2.

For the target past-participles, the participants produced a similar number of errors (281) as in Study 1 . As in Study 1 , the correctness of a response could not be predicted given the realization the participant had heard for that past-participle in the familiarization phase or by its duration in the lexical decision experiment.

For the analysis of the response latencies, the dataset did not include the 12 latencies that differed more than three standard deviations from the mean $(1322 \mathrm{~ms})$. The data were analyzed again by means of a multilevel regression model with as dependent variable the $\log$ of the response latency, and as random effects the participant and the past participle. The fixed effect predictors were also again the response given by the participant ("yes" or "no"), the duration of the stimulus (again logged), and most Familiarization type.

After removal of the outliers, the logged duration of the word showed an interaction with the response $(F(1,747)=$ $4.091, \mathrm{p}<0,05)$. Participants reacted more slowly to longer past-participles when producing errors. There was no main effect or an interaction of Familiarization type $(\mathfrak{p}>0.1)$ Participants reacted as quickly when they had heard the pastparticiple as unreduced in the familiarization phase (mean: $1330 \mathrm{~ms})$ as when they had heard it as reduced $(1328 \mathrm{~ms}$ )

In conclusion, participants did not react more quickly to unreduced past participles if they had heard these same pastparticiples also as unreduced in the familiarization phase. The unreduced and the reduced pronunciation variants appear to prime unreduced pronunciations equally well. This suggests that upon hearing a new word produced with a reduction that occurs for other words as well, participants reconstruct the unreduced form, and store both this unreduced form and the perceived reduced form in their mental lexicons.

\section{Conclusions}

This study addressed the question of how listeners process pronunciation variants following general reduction patterns. Several psycholinguistic models assume that the comprehension of variants is based on the word's unreduced pronunciation, which is stored in the mental lexicon [2]. Other models [3] assume that the mental lexicon may contain severa pronunciation variants for each word, which may be used in speech processing. This study investigated the roles of both mechanisms by means of a word learning task.

If lexical representations for pronunciation variants play an important role in speech processing, listeners should be sensitive to word-specific pronunciation variation. They are expected to store the reduced pronunciation variants they are presented with, even if the corresponding unreduced pronunciations can easily be deduced. Study 1 showed that this is indeed the case. If participants are presented with new words, half of which produced with a reduction that also occurs in other words, and a week later they hear all these words with this reduction in a lexical decision experiment, they react more quickly to the words that they heard as reduced also in the familiarization phase. Since participants heard different tokens in the lexical decision experiment than in the familiarization phase, this effect cannot be ascribed to just the mapping of the acoustic input onto lexical representations of this same input. This is especially clear since the reduced tokens presented in the lexical decision experiment were much longer than the reduced tokens presented in the familiarization phase, and even longer than the unreduced forms presented in that phase. Rather, the participants matched the acoustic input onto phonologically/phonetically similar tokens or abstract lexical representations reflecting the input in the familiarization phase.

Study 2 investigated whether there was also some role for reconstruction, in addition to the storage of the heard pronunciation variants. In this study, the main lexical decision experiment contained all target past-participles in their unreduced forms. If during the familiarization phase, participants formed lexical representations only for the pronunciation variants they heard, they would have been faster in reacting to the past-participles that they had heard as unreduced also in the familiarization phase. This appeared not to be the case. Participants reacted as quickly whether they had heard the past-participle before as reduced or unreduced. This finding suggests that listeners reconstruct the unreduced forms on the basis of the reduced pronunciation variants. It supports earlier work suggesting that the unreduced pronunciation has a privileged status in the comprehension of reduced speech [11].

Together the two studies thus suggest that upon hearing a new word with a regular reduction, listeners store the presented form, but also reconstruct the unreduced form. Both the storage of pronunciation variants and reconstruction play importan roles in speech processing. These data therefore support hybrid models of speech processing, assigning roles to both mechanisms [12]

Following [7], a control lexical decision experiment investigated whether the participants had incorporated in their mental lexicons the words presented to them in the familiarization phase. If they had, these words should hinder recognition of low frequency lexical neighbors. The control experiment did not show such inhibition. Nevertheless, there is evidence that the participants had stored the new pastparticiples, since in the main lexical decision experiment of Study 1 they reacted more quickly to the past-participles that they had heard as reduced also in the familiarization phase Apparently, the control experiment was not sufficiently sensitive. Further research is necessary into which differences between the experiments presented here and in [7] are responsible for the difference in sensitivity. One possibility is that the participants in the current study were less aware of the similarities between the new words and the neighbors since these new words were relatively short (with a monosyllabic stem) compared to words like cathedruke tested in [7], and since the segment that distinguished the new word from the existing neighbor was located rather late in some words [7].

The control lexical decision experiment also showed that participants were very careful with pseudo words. They appeared to think, based on the familiarization phase, that the complete series of experiments focused on pseudo words. This finding raises the question to which extent tasks effects may play a role in the present studies.

In conclusion, this study strongly suggests that both the storage of pronunciation variants and the reconstruction of unreduced pronunciations play roles in the learning of new words. Further research is necessary to obtain detailed insights in the relevance of these mechanisms for more general speech processing.

\section{Acknowledgements}

This research was supported by a European Young Investigator award from the European Science Foundation. In addition, I would like to thank Wies de Beijer for carrying out part of the experiments, and Lou Boves for his comments on an earlier version of this paper.

\section{References}

[1] Ernestus, M., Voice assimilation and segment reduction in casual Dutch: A corpus-based study of the phonology-phonetics interface, LOT, 2000

[2] Gaskel, G., "Modelling regressive and progressive effects of assimilation in speech perception", Journal of Phonetics 31: 447-463, 2003.

[3] Johnson, K., "Massive reduction in conversational American English", in K. Yoneyama and K. Maekawa [Eds.], Spontaneous speech: data and analysis, 29-54. The National International Institute for Japanese Language, 2004

[4] McLennan, C.T. Luce, P.A.,, and Charles-Luce, J., Psychology: Learning, Memory, and Cognition 29: 539-553, 2003.

[5] Mattys, S. L. and Liss, J. M., "On building models of spokenword recognition: When there is as much to learn from natura "oddities" as artificial normality", Perception \& Psychophysics 70: $1235-1242,2008$

6] Ranbom, L.J., and Connine, C.M. "Lexical representation of phonological variation in spoken word recognition", JML 57: 273-29, 2007 .

[7] Gaskell, M.G. and Dumay, N., "Phonological variation and inference in lexical access", JEP HPP 22: 144-158.

[8] Lively, S.E., Logan, J.S., and Pisoni, D.B., "Training Japanese listeners to identify English / $\mathrm{r} /$ and /1/: II. The role of phonetic environment and talker variability in learning new perceptual categories", JASA 94: 1242-1255, 1993.

[9] Baayen, R.H., Davidson, D.J. and Bates, D.M., "Mixed-effects modeling with crossed random effects for subjects and items", JML 59: 390-412, 2008

[10] Jaeger, T.F., "Categorical data analysis: Away from ANOVA (transformation or not) and towards logit mixed models", JML 59: 434-446, 2008.

[11] Ernestus, M., Baayen, R.H., and Schreuder, R., "The recognition of reduced word forms", Brain and Language: 81 , 162-173, 2002.

[12] Hawkins, S. "Roles and representations of systematic fine phonetic detail in speech understanding", Journal of Phonetics 31:373-405, 2003 . 